\title{
TITLE:
}

\section{Ionization degree for strong evaporation of metals}

$\operatorname{AUTHOR}(S)$ :

Gusarov, Andrey V.; Aoki, Kazuo

CITATION:

Gusarov, Andrey V. ... [et al]. Ionization degree for strong evaporation of metals. PHYSICS OF PLASMAS 2005, 12(8): 083503.

ISSUE DATE:

2005-08

URL:

http://hdl.handle.net/2433/39795

\section{RIGHT:}

Copyright 2005 American Institute of Physics. This article may be downloaded for personal use only. Any other use requires prior permission of the author and the American Institute of Physics. 


\title{
lonization degree for strong evaporation of metals
}

\author{
Andrey V. Gusarov ${ }^{\text {a) }}$ \\ Baikov Institute of Metallurgy, Russian Academy of Sciences, Leninsky Prospect 49, 119991 Moscow, Russia \\ Kazuo Aoki ${ }^{\mathrm{b})}$ \\ Department of Aeronautics and Astronautics, Graduate School of Engineering, Kyoto University, \\ Kyoto 606-8501, Japan
}

(Received 19 May 2005; accepted 29 June 2005; published online 12 August 2005)

\begin{abstract}
Kinetic equations for ions and neutrals are numerically solved in the plasma sheath formed at a condensed phase when strong evaporation is taking place. The Boltzmann distribution is assumed for electrons. A weakly ionized vapor with the Debye length much shorter than the mean free path is considered. This is typical for laser evaporation of metals. Under these conditions, the sheath consists of a Knudsen layer and a thin charge separation layer between the Knudsen layer and the condensed phase. The self-consistent electrostatic field in the Knudsen layer is obtained from the quasineutrality condition. The potential barrier in the charge separation layer is determined by the charge balance. Kinetic boundary conditions for neutrals and charges are estimated by the detailed balance principle from the parameters of the saturated vapor. The transport of charges in the sheath is controlled by ions and depends on ion-neutral collisions and the self-consistent electrostatic field. Ionization degree in the vapor formed by strong evaporation increases with the Mach number and can attain values about $30 \%$ higher than the ionization degree in the saturated vapor. Two factors contribute to this increase. The first is the drop of the potential barrier in the charge separation layer and the second is the strengthening of the field in the Knudsen layer. The ionization equilibrium may be disturbed by a considerable excess of charges. (C) 2005 American Institute of Physics.
\end{abstract}

[DOI: 10.1063/1.2002229]

\section{INTRODUCTION}

Radiation absorption in vapor has a dramatic effect on pulsed laser evaporation of metals. Laser radiation is absorbed on the surface and causes thermal evaporation. The vapor can absorb due to inverse bremsstrahlung on free electrons and photoionization of exited atoms. ${ }^{1}$ This absorption is, generally, weak because the ionization degree and the fraction of excited atoms are low at typical vapor temperatures below $1 \mathrm{eV}$. However, as the energy of photons is transferred directly to electrons, the electron temperature may become high enough to initiate intensive avalanche ionization by electron impact. This process, referred to as optical breakdown, turns the vapor to a highly ionized and highly absorptive state. A so-called radiation-supported detonation wave is formed, ${ }^{2}$ and the vapor shields the surface from radiation. The optical breakdown reduces material removal and increases the energy of ejected species. The first is not desirable for laser machining ${ }^{3}$ but the second can be useful for pulsed laser deposition. ${ }^{4}$

Stability of the low-absorbing vapor flow is controlled by the balance of electron thermal energy gained by radiation absorption and lost due to collisions with colder heavy species and, probably, due to thermal conduction to the surface. Theoretical estimate ${ }^{5}$ confirms that kinetics of optical breakdown is very sensitive to the initial density of absorbing species. Therefore, even weak initial ionization is important at laser evaporation. Such fast processes are usually far from

\footnotetext{
${ }^{a)}$ Electronic mail: AV.Gusarov@ @relcom.ru

${ }^{\text {b)} E l e c t r o n i c ~ m a i l: ~ a o k i @ a e r o . m b o x . m e d i a . k y o t o-u . a c . j p ~}$
}

ionization equilibrium, so that the ionization degree is determined by transport of electrons, ions, and neutrals in the collisional sheath formed near the surface.

Known kinetic approaches to the collisional sheath ${ }^{6-11}$ are generally concerned with a cold catalytic wall without net flux of neutrals and describe processes similar to ambipolar diffusion. On the other hand, strong evaporation is studied for neutral gas only. ${ }^{12-22}$ At strong evaporation of neutral gas, the velocity distribution of molecules ejected from the surface considerably differs from the distribution of incident molecules. This discontinuity in the distribution function indicates high nonequilibrium near the evaporating surface that disappears in a few mean free paths downstream.

Early analytical ${ }^{12-14}$ and numerical ${ }^{15}$ approaches to the nonequilibrium Knudsen layer at evaporation gave rather precise estimates of vapor flow parameters. It is convenient to specify the equilibrium vapor flow far from the Knudsen layer by pressure, $p / p_{s}$, and temperature, $T / T_{s}$, ratios and Mach number $M=u / c$ with $p, T, u$, and $c$ being vapor pressure, temperature, flow velocity, and local sound speed, respectively, and $T_{s}$ surface temperature and $p_{s}$ saturated vapor pressure at this temperature. Possible vapor states were found to lay on a universal curve in the three-dimensional space of these dimensionless parameters with the Mach number in the interval $0 \leqslant M \leqslant 1$. These results were recently confirmed by direct simulation Monte Carlo (DSMC), ${ }^{16} \mathrm{nu}-$ merical approaches to the Bhatnagar-Gross-Krook (BGK) model of the collision operator in the Boltzmann equation, ${ }^{17,18}$ and rigorous analysis of the Boltzmann 
equation. ${ }^{19-22}$ Detailed velocity distribution function inside the Knudsen layer is obtained. ${ }^{18}$

When several types of molecules are ejected from the surface, the vapor enriches with the lighter components relative to the composition of the saturated vapor because of higher thermal velocities of the lighter molecules. ${ }^{23,24}$ Therefore, one can expect faster electron transport through the Knudsen layer in case of electron emission. However, this effect should be less than in a mixture of neutrals because charge separation leads to a strong electrostatic field decelerating the ejected electrons and accelerating ions that tends to equate the densities of electrons and ions in a distance about the Debye length. This indicates the importance of the self-consistent electrostatic field characteristic for the plasma sheath.

\section{SATURATED VAPOR}

The variety of plasma parameters makes it difficult to propose a universal model, so we first consider weak evaporation at $M \ll 1$ when ionized vapor is approximately in equilibrium with the condensed phase. This gives an estimate of the domain of possible plasma parameters at strong evaporation, $M \sim 1$. Figure 1 (a) shows the temperature dependences of pressure $p_{s}$ and ionization degree $\alpha_{s}$ of saturated vapors of aluminum and copper calculated using thermodynamic tables. ${ }^{25,26}$ One can see that the ionization degree is small compared to unity at least up to the temperature of $T$ $=4500 \mathrm{~K}$.

The distribution functions of vapor species over molecular velocity c are Maxwell distributions $f_{M \beta}\left(n_{\beta}, 0, T\right)$ with subscript $\beta=n, i$, and $e$ for neutrals, ions, and electrons, respectively, and $n_{n}=p_{s} /(k T)$ and $n_{i}=n_{e}=\alpha_{s} n_{n}$. Here, the Maxwell distribution is specified by density $n$, flow velocity $\mathbf{u}$, and temperature $T$ :

$$
f_{M \beta}(n, \mathbf{u}, T)=n\left(\frac{m_{\beta}}{2 \pi k T}\right)^{3 / 2} \exp \left(-\frac{m_{\beta}(\mathbf{c}-\mathbf{u})^{2}}{2 k T}\right),
$$

where $m_{\beta}$ is the molecular mass and $k$ the Boltzmann constant. According to the detailed balance principle, the distribution of neutrals ejected from the surface at equilibrium should be equal to Maxwellian (1) at positive normal component of molecular velocity $c_{z}>0$ (let axis $\mathrm{Z}$ be directed along the external normal). This distribution complements the half-Maxwellian $f_{M n}, c_{z}<0$, of neutrals striking the surface to the equilibrium function (1). However, similar estimates for distributions of ejected charges, ions and electrons, are not valid because the surface generally acquires an electric charge and the electric field formed in the adjacent sheath layer highly influences the transport of charges.

The charge balance in the sheath can be estimated by the flux of electrons coming from the plasma to the external edge of the sheath,

$$
F_{e}^{-}=-\int_{c_{z}<0} f_{M e}\left(n_{e}, 0, T\right) c_{z} \mathrm{~d} \mathbf{c}=n_{e} \sqrt{\frac{k T}{2 \pi m_{e}}},
$$

and thermionic flux $F_{e}^{+}$at the surface as shown in Fig. 2(a). Thermal emission of electrons is given by the RichardsonDushman equation: ${ }^{27,28}$

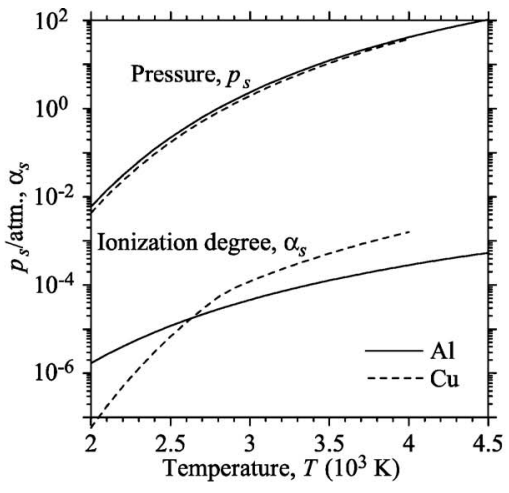

(a)

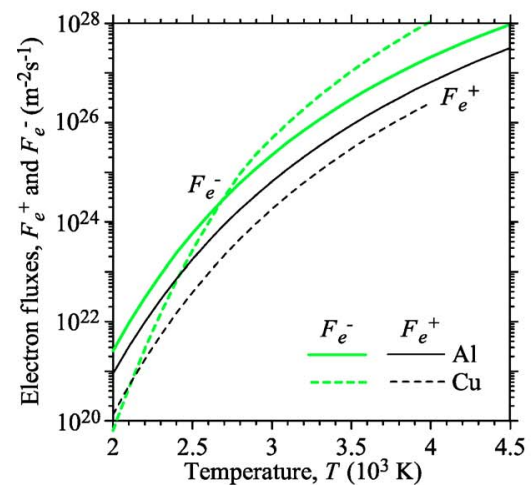

(b)

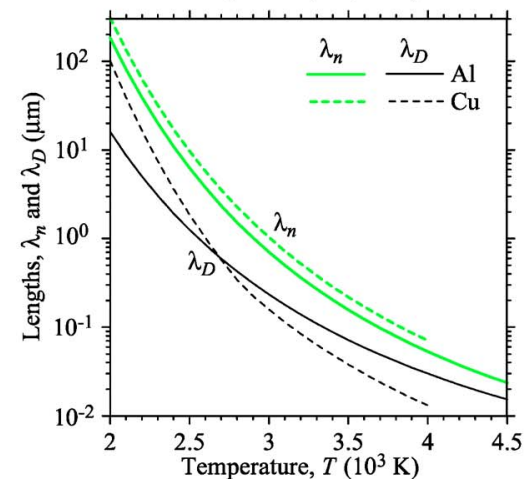

(c)

FIG. 1. (Color online). Vapor-condensed phase equilibrium parameters for $\mathrm{Al}$ and $\mathrm{Cu}$ : (a) saturated vapor pressure $p_{s}$ and ionization degree $\alpha_{s}$; (b) thermionic $F_{e}^{+}$and backward $F_{e}^{-}$fluxes of electrons; (c) Debye length $\lambda_{D}$ and mean free path of neutrals $\lambda_{n}$.

$$
F_{e}^{+}=\frac{4 \pi m_{e}(k T)^{2}}{h^{3}} \exp \left(-\frac{W}{k T}\right),
$$

where $h$ is the Planck's constant and $W$ the work function. Figure 1(b) shows these fluxes for $\mathrm{Al}$ and $\mathrm{Cu}$ vapors at equi- (a)

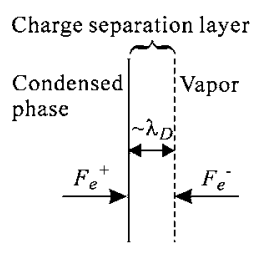

(b)

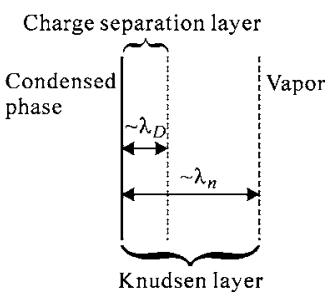

FIG. 2. Sheath structure at equilibrium (a) and at evaporation (b): thermionic $F_{e}^{+}$and backward $F_{e}^{-}$electron fluxes are shown by arrows in $(\mathrm{a}) ; \lambda_{D}$ is the Debye length and $\lambda_{n}$ the mean free path of neutrals. 
TABLE I. Parameters of $\mathrm{Al}$ and $\mathrm{Cu}$.

\begin{tabular}{ccccc}
\hline \hline Name & Symbol & Unit & $\mathrm{Al}$ & $\mathrm{Cu}$ \\
\hline Work function $^{\mathrm{a}}$ & $W$ & $\mathrm{eV}$ & 4.18 & 4.51 \\
Atomic diameter $^{\mathrm{a}}$ & $d$ & $\mathrm{~nm}$ & 0.282 & 0.2551 \\
$\begin{array}{c}\text { Charge exchange } \\
\text { Cross-section }\end{array}$ & $\sigma_{e x}$ & $10^{-18} \mathrm{~m}^{2}$ & 1.9 & 1.6 \\
Cross-section ratio & $\sigma_{e x} / \pi d^{2}$ & - & 7.6 & 7.8 \\
\hline \hline
\end{tabular}

${ }^{\mathrm{a}}$ Reference 29.

${ }^{\mathrm{b}}$ At $1 \mathrm{eV}$ (Ref. 31).

librium with their condensed phases. The values of work function are taken from Ref. 29 and listed in Table I. One can see that the typical case is when $F_{e}^{+}<F_{e}^{-}$(Al and $\mathrm{Cu}$ at $T$ $>2100 \mathrm{~K})$. In such conditions, the condensed phase is charged negatively and an electrostatic field arises in the sheath that partly repels electrons coming from the vapor to equate their flux at the surface to the thermionic flux. The sheath thickness at equilibrium is estimated as the Debye length

$$
\lambda_{D}=\sqrt{\frac{k T}{4 \pi e^{2} n_{e}}},
$$

where $e$ is the elementary charge.

When evaporation starts, a nonequilibrium Knudsen layer arises with the thickness of about several mean free paths of neutrals: ${ }^{14-18}$

$$
\lambda_{n}=1 /\left(\pi d^{2} n\right),
$$

where $d$ is the atomic diameter listed in Table I. Figure 1(c) shows that the Debye length is, generally, several times less than the mean free path, so that the charge separation layer is well inside the Knudsen layer as shown in Fig. 2(b) and sheath thickness at evaporation is determined by mean free path $\lambda_{n}$.

\section{MODEL}

Further consideration is restricted to weakly ionized vapor when ionization degree is so small that collisions between charges are negligible. Ionization/recombination reactions in the sheath are also neglected according to previous estimates. $^{7-11}$ Only the limiting case of infinitely small ratio $\lambda_{D} / \lambda_{n}$ is studied. According to the above examples, the last assumption is not rigorous but a reasonable approximation. In this limit, the sheath [see Fig. 2(b)] can be regarded as a quasineutral Knudsen layer with boundary conditions determined by thin collisionless charge separation layer. Negative electrostatic potential $\phi_{s}<0$ of the condensed phase relative to the bulk of the vapor is assumed, so that electric field in the charge separation layer reduces ion emission.

\section{A. Kinetic equations}

A coordinate system is introduced with the origin on the surface and axis $Z$ directed along the external normal to the condensed phase. Both the surface and the charge separation layer lay in plane $z=0$. To avoid ambiguity, values at $z=0$ will be referred to the boundary between the charge separa- tion layer and the Knudsen layer and values on the boundary between the condensed phase and the charge separation layer will be designated by subscript $s$. Velocity distribution functions of heavy species, neutrals, $f_{n}$, and ions, $f_{i}$, are described by Boltzmann equations in domain $z>0$ :

$$
\begin{aligned}
& \frac{\partial f_{n}}{\partial t}+c_{z} \frac{\partial f_{n}}{\partial z}=J_{n n}, \\
& \frac{\partial f_{i}}{\partial t}+c_{z} \frac{\partial f_{i}}{\partial z}-\frac{e}{m_{i}} \frac{\partial \phi}{\partial z} \frac{\partial f_{i}}{\partial c_{z}}=J_{i n},
\end{aligned}
$$

where $t$ is the time, $\phi$ the electrostatic potential, and $J_{n n}$ and $J_{\text {in }}$ the neutral-neutral and ion-neutral collision integrals, respectively. Collisions between neutrals and charges are neglected in Eq. (6) because of small ionization degree.

High atom-to-electron mass ratio is known to result in three significantly different relaxation times $\tau_{e e}, \tau_{n n}$, and $\tau_{e n}$ for electron-electron, heavy-heavy, and electron-heavy species collisions, respectively, which are in proportion: ${ }^{30}$

$$
\tau_{e e}: \tau_{n n}: \tau_{e n}=1:\left(m_{n} / m_{e}\right)^{1 / 2}:\left(m_{n} / m_{e}\right) .
$$

At flow velocity about the sound speed, vapor passes through the Knudsen layer with thickness about several mean free paths $\lambda_{n}$ for a time about the intermediate relaxation time $\tau_{n n}$. This means that electrons are in equilibrium with themselves in the Knudsen layer and, on the other hand, collisions with heavy species do not influence them. Intensive thermionic emission [see Fig. 1(b)] ensures electronic temperature to be about the temperature of the condensed phase $T_{s}$, so that Maxwell velocity distribution $f_{M e}\left(n_{e}, 0, T_{s}\right)(1)$ is a good approximation. The Boltzmann distribution is accepted for electron density:

$$
n_{e}(z)=n_{e}(\infty) \exp \left(e \phi / k T_{s}\right),
$$

where electrostatic potential $\phi$ is meant to be zero at infinity.

The same approach for electrons was used in Refs. 7-9 while numerical solution of kinetic equations for electrons in Refs. 10 and 11 indicated that Eq. (9) is rather rough. However, the characteristic time for the diffusion-controlled sheath considered in these works should be significantly higher than $\tau_{n n}$ and may approach $\tau_{e n}$. So, electron collisions with heavy specious do disturb the Boltzmann distribution (9) in these conditions. Approximation (9) is expected to be more precise in the conditions of strong evaporation.

Electrostatic potential can be excluded from Eq. (7) using Eq. (9) and the quasineutrality condition

$$
n_{i}=n_{e}
$$

that results in the following kinetic equation for ions:

$$
\frac{\partial f_{i}}{\partial t}+c_{z} \frac{\partial f_{i}}{\partial z}-\frac{k T_{s}}{m_{i} n_{i}} \frac{\partial n_{i}}{\partial z} \frac{\partial f_{i}}{\partial c_{z}}=J_{i n} .
$$

Collision integral $J_{\text {in }}$ in the right-hand side of Eq. (11) depends on the distribution function of neutrals, which can be found from Eq. (6). There is no explicit interaction with electrons, however, electrons participate in formation of the electrostatic field taken into account through the third term in the left-hand side of Eq. (11). Similar kinetic formulation was 
used for the plasma sheath earlier. ${ }^{10,11}$ The principal difference of the present work is the highly nonequilibrium velocity distribution of neutrals typical for strong evaporation ${ }^{18}$ while only Maxwell distribution of neutrals was studied in Refs. 10 and 11.

The problem of strong evaporation is known to be not sensitive to the character of interatomic interaction, and so the relaxation model of the collision integral gives a good approximation. ${ }^{17,18}$ This model is implemented here for neutral-neutral collisions:

$$
J_{n n}=\omega_{n n}\left(f_{M n}\left(n_{n}, \mathbf{u}_{n}, T_{n}\right)-f_{n}\right),
$$

with standard definition of distribution function moments:

$$
\begin{aligned}
& n_{\beta}=\int f_{\beta} \mathrm{d} \mathbf{c}, \quad 0=\int \mathbf{C}_{\beta} f_{\beta} \mathrm{d} \mathbf{c}, \\
& \mathbf{P}_{\beta}=m_{\beta} \int \mathbf{C}_{\beta} \mathbf{C}_{\beta} f_{\beta} \mathrm{d} \mathbf{c}, \quad \mathbf{q}_{\beta}=\left(m_{\beta} / 2\right) \int C_{\beta}^{2} \mathbf{C}_{\beta} f_{\beta} \mathrm{d} \mathbf{c}, \\
& n_{\beta} k T_{\beta}=p_{\beta}=\left(P_{x x \beta}+P_{y y \beta}+P_{z z \beta}\right) / 3,
\end{aligned}
$$

where $\mathbf{u}_{\beta}$ is the flow velocity, $\mathrm{C}_{\beta}=\mathbf{c}-\mathbf{u}_{\beta}$ the peculiar velocity, $\mathbf{P}_{\beta}$ the pressure tensor, $\mathbf{q}_{\beta}$ the heat flow vector, $p_{\beta}$ the hydrostatic pressure, and $T_{\beta}$ the temperature. Relaxation frequency is estimated as ${ }^{18}$

$$
\omega_{n n}=\frac{32}{15} n_{n} d^{2}\left(\frac{\pi k T_{n}}{m_{n}}\right)^{1 / 2},
$$

where $d$ is the atomic diameter given in Table $\mathrm{I}$ for $\mathrm{Al}$ and $\mathrm{Cu}$.

The most important process of ion-neutral interaction is known to be charge exchange. Resonant charge-exchange cross-sections $\sigma_{e x}$ are calculated in Ref. 31 and listed in Table I for $\mathrm{Al}-\mathrm{Al}^{+}$and $\mathrm{Cu}-\mathrm{Cu}^{+}$collisions at relative energies of about $1 \mathrm{eV}$. The ratio of charge exchange to elastic collision cross-section $\pi d^{2}$ shown in the last row of Table I is large. Therefore, elastic collisions are neglected. In the approximation of linear trajectory, charge exchange is equivalent to a head-on collision ${ }^{6}$ that gives the following collision term:

$$
J_{i n}=\int\left[f_{i}\left(\mathbf{c}^{\prime}\right) f_{n}(\mathbf{c})-f_{i}(\mathbf{c}) f_{n}\left(\mathbf{c}^{\prime}\right)\right] \sigma_{e x} g \mathrm{~d} \mathbf{c}^{\prime},
$$

where $\mathbf{g}=\mathbf{c}-\mathbf{c}^{\prime}$ is the relative velocity. Cross-section $\sigma_{e x}$ generally depends on $g$, however, calculations ${ }^{31}$ in the range of relative energies from 0.1 to $10 \mathrm{eV}$ indicate that this dependence is weak. Therefore, the constant value of this crosssection estimated at $1 \mathrm{eV}$ is accepted in this work (see Table I).

\section{B. Boundary conditions}

Neutrals, ions, and electrons incident to the condensed phase are supposed to stick the surface without reflection. In this case, the velocity distributions of ejected species are independent of the distributions of incident species and are the same as would be at equilibrium at the temperature of condensed phase $T_{s}$. The distributions ejected from the sur- face at equilibrium are estimated from Eq. (1) by the principle of detailed balance. This gives a well-known result for neutrals: ${ }^{12-22}$

$$
f_{n}(0)=f_{M n}\left(n_{s}, 0, T_{s}\right) \quad \text { at } c_{z}>0,
$$

where $n_{s}=p_{s} / k T_{s}$, is the saturated vapor density.

The electrostatic barrier of the charge separation layer complicates application of the same method to charges. A relation like Eq. (17) is valid for ions at the boundary between the condensed phase and the charge separation layer while Boltzmann equation (11) requires to specify distribution of ions $f_{i}(0)$ passed through the potential barrier. This gives an additional Arrhenius factor:

$$
f_{i}(0)=\exp \left(\frac{e\left[\phi_{s}-\phi(0)\right]}{k T_{s}}\right) f_{M i}\left(n_{i s}, 0, T_{s}\right) \quad \text { at } c_{z}>0,
$$

where difference $\phi_{s}-\phi(0)$ is assumed to be negative and $n_{i s}$ is an unknown function of temperature. According to the detailed balance principle, distribution (18) is equal to (1) at equilibrium:

$$
f_{M i}\left(\alpha_{s} n_{s}, 0, T_{s}\right)=\exp \left(\frac{e \phi_{s}^{e q}}{k T_{s}}\right) f_{M i}\left(n_{i s}, 0, T_{s}\right)
$$

where $\alpha_{s}$ is the ionization degree of saturated vapor and $\phi_{s}^{e q}<0$ the condensed phase potential at equilibrium. It is taken into account that $\phi(0)=0$ at equilibrium because there is no Knudsen layer. One can exclude $n_{i s}$ from Eqs. (18) and (19):

$$
f_{i}(0)=\exp \left(\frac{e\left[\phi_{s}-\phi_{s}^{e q}-\phi(0)\right]}{k T_{s}}\right) f_{M i}\left(\alpha_{s} n_{s}, 0, T_{s}\right) \quad \text { at } c_{z}>0 .
$$

The last equation indicates that the boundary condition for ions depends on their density in saturated vapor $\alpha_{s} n_{s}$ as well as on the change of potential barrier relative to equilibrium.

Condensed phase potential $\phi_{s}$ can be estimated from the charge balance at the surface. Note that in the conditions of intensive thermionic emission [see Fig. 1(b)], contribution of ions into charge balance is negligible because of low ratio of ion to electron thermal velocity. Consider electron balance at the boundary between the condensed phase and the charge separation layer. Electrostatic field in the charge separation layer partly repels electrons coming from the Knudsen layer, therefore electron flux in negative direction given by Eq. (2) is multiplied by an Arrhenius factor similar to that in Eq. (18):

$$
F_{e}^{+}=n_{e}(0) \sqrt{\frac{k T_{s}}{2 \pi m_{e}}} \exp \left(\frac{e\left[\phi_{s}-\phi(0)\right]}{k T_{s}}\right),
$$

where $F_{e}^{+}$is the thermionic flux given by the RichardsonDushman equation (3). The similar balance at equilibrium is

$$
F_{e}^{+}=\alpha_{s} n_{s} \sqrt{\frac{k T_{s}}{2 \pi m_{e}}} \exp \left(\frac{e \phi_{s}^{e q}}{k T_{s}}\right) .
$$

The flux $F_{e}^{+}$can be excluded from Eqs. (21) and (22) to obtain a relative potential barrier in the charge separation layer: 


$$
\frac{e\left[\phi_{s}-\phi_{s}^{e q}-\phi(0)\right]}{k T_{s}}=\ln \left(\frac{\alpha_{s} n_{s}}{n_{i}(0)}\right)
$$

where electron density $n_{e}(0)$ at the boundary between the charge separation and Knudsen layers is replaced by $n_{i}(0)$ according to Eq. (10). Substitution of Eq. (23) into Eq. (20) results in boundary condition for ions:

$$
f_{i}(0)=f_{M i}\left(\frac{\left(\alpha_{s} n_{s}\right)^{2}}{n_{i}(0)}, 0, T_{s}\right) \quad \text { at } c_{z}>0 .
$$

This condition does not specify the distribution of ejected species explicitly as in the case of neutrals [see Eq. (17)] but only connects it with zero-order moment $n_{i}(0)$.

Far from the surface, strong evaporation produces uniform flow of equilibrium vapor with one degree of freedom, ${ }^{17-24}$ so that one flow parameter should be specified at infinity and steady-state values of other parameters can be found from the kinetic equations. This relation between vapor flow parameters is known as gas-dynamic boundary condition of strong evaporation, which has been extensively studied for one-component gas. ${ }^{13-22}$ In this work we specify pressure at infinity $p_{n}(\infty)$. The functions of flow velocity $u_{z n}(\infty)=u_{z i}(\infty)$ and temperature $T_{n}(\infty)=T_{i}(\infty)$ versus pressure are the same as at one-component evaporation. The objective of the present study is to calculate ionization degree $\alpha(\infty)$ $=p_{i}(\infty) / p_{n}(\infty) \ll 1$.

\section{NUMERICAL METHOD}

Vapor parameters change very sharply near the condensed phase and gradually tend to constants with increasing the distance $z$ to the surface. Therefore high spatial resolution is required at small $z$ while it becomes excessive at large $z$. Such problems were numerically solved on nonuniform spatial grids ${ }^{17}$ or with high-order schemes. ${ }^{18}$ In this work, a nonlinear coordinate transform to dimensionless variable $w$ is used:

$$
(1+w)^{2}=1+z / a
$$

with transform parameter $a$, which enlarges the region near the surface and condenses space at infinity. Dimensionless variables are introduced as shown in Table II: $\tau$ and $\xi$ are dimensionless time and molecular velocity, respectively, and dimensionless velocity distribution functions and their moments are denoted by waves. Unknown value $n_{i}(0)$ is used in the definition of dimensionless velocity distribution function $f_{i}$ of ions and its moments (see Table II). This is possible because kinetic equation for ions (11) is invariant when $f_{i}$ is multiplied by a factor, and so the resulting dimensionless problem becomes independent of $n_{i}(0)$.

Kinetic equation for neutrals (6) with collision term (12) and relaxation frequency (15) becomes
TABLE II. Dimensionless variables.

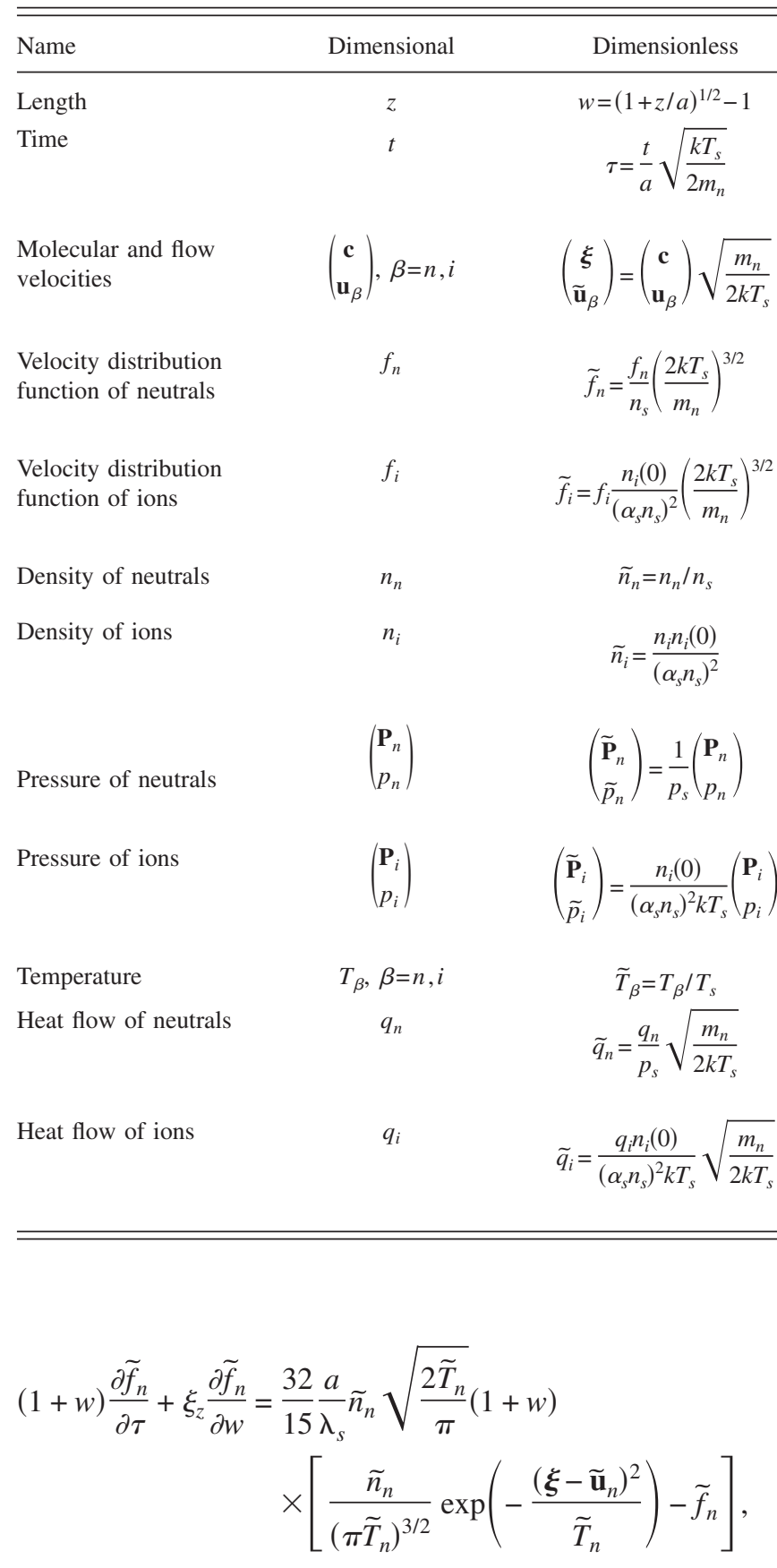

where

$$
\lambda_{s}=1 /\left(\pi d^{2} n_{s}\right),
$$

is the mean free path of neutrals in the saturated vapor at the temperature of condensed phase. Kinetic equation for ions (11) with collision term (16) is transformed to

$$
\begin{aligned}
&(1+w) \frac{\partial \tilde{f}_{i}}{\partial \tau}+\xi_{z} \frac{\partial \tilde{f}_{i}}{\partial w}-\frac{1}{2 \tilde{n}_{i}} \frac{\partial \widetilde{n}_{i}}{\partial w} \frac{\partial \tilde{f}_{i}}{\partial \xi_{z}} \\
&=2 \frac{a}{\lambda_{s}} \frac{\sigma_{e x}}{\pi d^{2}}(1+w) \int\left[\tilde{f}_{i}\left(\boldsymbol{\xi}^{\prime}\right) \tilde{f}_{n}(\boldsymbol{\xi})-\tilde{f}_{i}(\boldsymbol{\xi}) \tilde{f}_{n}\left(\boldsymbol{\xi}^{\prime}\right)\right]\left|\boldsymbol{\xi}-\boldsymbol{\xi}^{\prime}\right| \mathrm{d} \boldsymbol{\xi}^{\prime} .
\end{aligned}
$$

Boundary conditions at the surface (17) and (24) reduce to 


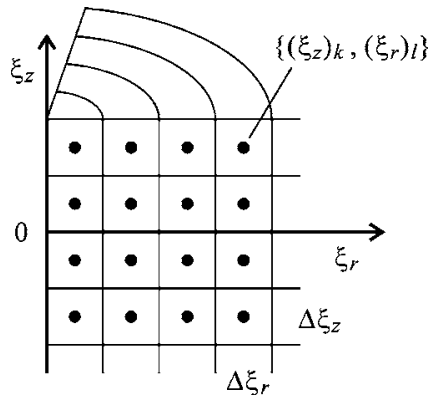

FIG. 3. Cylindrical coordinates $\left(\xi_{z}, \xi_{r}\right)$, in the velocity space and its discretization: $\Delta \xi_{z}$ and $\Delta \xi_{r}$ are cell dimensions and $\left(\xi_{z}\right)_{k}$ and $\Delta\left(\xi_{r}\right)_{l}$ node coordinates.

$$
\tilde{f}_{n}(0)=\tilde{f}_{i}(0)=\frac{1}{\pi^{3 / 2}} e^{-\xi^{2}} \text { at } \xi_{z}>0,
$$

and pressure ratio $\widetilde{p}_{n}(\infty)=p_{n}(\infty) / p_{s}$ is specified at infinity.

A discrete velocity method described below reduces Eqs. (26) and (28) to a set of advection equations, which are solved by a second-order finite volume method. ${ }^{18}$ In the considered evaporation problem, distribution functions are symmetric in the velocity space about the normal to the surface. Therefore, molecular velocity $\boldsymbol{\xi}$ is specified by its axial $\xi_{z}$ and radial $\xi_{r}$ components in the cylindrical coordinate system shown in Fig. 3. Calculation domain $\left\{\Xi_{z}^{-}<\xi_{z}<\Xi_{z}^{+}, 0<\xi_{r}\right.$ $\left.<\Xi_{r}\right\}$ is divided into $N_{z} \times N_{r}$ rectangular toroidal cells with sides $\Delta \xi_{z}=\left(\Xi_{z}^{+}-\Xi_{z}^{-}\right) / N_{z}$ and $\Delta \xi_{r}=\Xi_{r} / N_{r}$, centers at $\left\{\left(\xi_{z}\right)_{k}\right.$ $\left.=\Xi_{z}^{-}+(k-1 / 2) \Delta \xi_{z},\left(\xi_{r}\right)_{l}=(l-1 / 2) \Delta \xi_{r}\right\}$ for $k=1 \ldots N_{z}$ and $l$ $=1 \ldots N_{r}$ (see Fig. 3), and volumes

$$
V_{l}=\int_{\left(\xi_{z}\right)_{k}-\Delta \xi_{z} / 2}^{\left(\xi_{z}\right)_{k}+\Delta \xi_{z} / 2} \mathrm{~d} \xi_{z} 2 \pi \int_{\left(\xi_{r}\right)_{l}-\Delta \xi_{l} / 2}^{\left(\xi_{r}\right)_{l}+\Delta \xi_{r} / 2} \xi_{r} \mathrm{~d} \xi_{r}=2 \pi\left(\xi_{r}\right)_{l} \Delta \xi_{z} \Delta \xi_{r}
$$

Discrete distribution function $\left(\tilde{f}_{\beta}\right)_{k l}$ is defined as the average of continuous distribution $\tilde{f}_{\beta}$ over cell $(k, l)$.

\section{A. Equation for neutrals}

Kinetic equation for neutrals (26) is approximated on this grid by

$$
\begin{aligned}
(1+w) \frac{\partial\left(\tilde{f}_{n}\right)_{k l}}{\partial \tau}+\left(\xi_{z}\right)_{k} \frac{\partial\left(\tilde{f}_{n}\right)_{k l}}{\partial w}= & \frac{32}{15} \frac{a}{\lambda_{s}} \widetilde{n}_{n} \sqrt{\frac{2 \widetilde{T}_{n}}{\pi}}(1+w) \\
& \times\left[\left(\tilde{f}_{M n}\right)_{k l}-\left(\tilde{f}_{n}\right)_{k l}\right],
\end{aligned}
$$

with discrete equilibrium distribution $\left(\widetilde{f}_{M n}\right)_{k l}$ and the following definition of moments:

$$
\begin{aligned}
& \widetilde{n}_{n}=\sum_{k, l=1}^{N_{z}, N_{r}}\left(\tilde{f}_{n}\right)_{k l} V_{l}, \\
& \tilde{n}_{n} \widetilde{u}_{n}=\sum_{k, l=1}^{N_{z}, N_{r}}\left(\xi_{z}\right)_{k} \tilde{f}_{k l} V_{l},
\end{aligned}
$$

$$
\frac{3}{2} \widetilde{n}_{n} \widetilde{T}_{n}=\sum_{k, l=1}^{N_{z}, N_{r}}\left\{\left[\left(\xi_{z}\right)_{k}-\widetilde{u}_{n}\right]^{2}+\left(\xi_{r}\right)_{l}^{2}\right\} \widetilde{f}_{k l} V_{l} .
$$

We do not distinguish here between $z$-component $\widetilde{u}_{n z}$ and absolute value $\tilde{u}_{n}$ of flow velocity because they are equal at the cylindrical geometry of the problem.

Approximation of discrete equilibrium distribution $\left(\widetilde{f}_{M n}\right)_{k l}$ by Maxwell function (1) taken at node $\left\{\left(\xi_{z}\right)_{k},\left(\xi_{r}\right)_{l}\right\}$ generally gives nonconservative numerical schemes, ${ }^{18,32}$ so that careful calculation thereof is required. It is known from statistical mechanics ${ }^{33}$ that the logarithm of the distribution function at equilibrium is an additive integral of motion, which is, actually, a linear combination of energy, momentum, and angular momentum. For ideal gas, this integral is equal to a quadratic form of molecular velocity that gives the following general expression for the discrete equilibrium distribution:

$$
\left(\tilde{f}_{M n}\right)_{k l}=A \exp \left(-\Gamma\left\{\left[\left(\xi_{z}\right)_{k}-\Delta\right]^{2}+\left(\xi_{r}\right)_{l}^{2}\right\}\right),
$$

with constants $\mathrm{A}, \Gamma$, and $\Delta$. To approximate Maxwell distribution (1), these constants should tend to $\mathrm{A}=\tilde{n}_{n}, \Gamma$ $=\widetilde{n}_{n} /\left(\pi \widetilde{T}_{n}\right)^{3 / 2}$, and $\Delta=\widetilde{u}_{n}$ when $\Delta \xi_{z}$ and $\Delta \xi_{r}$ tend to zero. Equations like (35) were applied in Refs. 18 and 32.

To obtain a conservative approximation of the collision term, the three unknowns can be found from discrete analogs of conservation laws:

$$
\sum_{k, l=1}^{N_{z}, N_{r}}\left[\left(\tilde{f}_{M n}\right)_{k l}-\left(\tilde{f}_{n}\right)_{k l}\right]\left(\begin{array}{c}
1 \\
\left(\xi_{z}\right)_{k} \\
\left(\xi_{z}\right)_{k}^{2}+\left(\xi_{r}\right)_{l}^{2}
\end{array}\right) V_{l}=0 .
$$

The resulting equations are solved by the Newton's method as explained in Ref. 18.

\section{B. Equation for ions}

The collision (right-hand side) term of Eq. (28) can be expressed as a sum of integrals over cells in the velocity space (see Fig. 3):

$$
\begin{aligned}
\tilde{J}_{i n}= & 2 \frac{a}{\lambda_{s}} \frac{\sigma_{e x}}{\pi d^{2}}(1+w) \sum_{k^{*}, l^{*}=1}^{N_{z}, N_{r}} \int_{\left(\xi_{z}\right) k^{*}-\Delta \xi_{z} / 2}^{\left(\xi_{z}\right) k^{*}+\Delta \xi_{z} / 2} \mathrm{~d} \xi_{z}^{\prime} \\
& \times \int_{\left(\xi_{r}\right)_{l^{*}}-\Delta \xi_{r} / 2}^{\left(\xi_{r}\right)^{*}+\Delta \xi_{r} / 2}\left[\tilde{f}_{i}\left(\xi_{z}^{\prime}, \xi_{r}^{\prime}\right) \tilde{f}_{n}\left(\xi_{z}, \xi_{r}\right)\right. \\
& \left.-\tilde{f}_{i}\left(\xi_{z}, \xi_{r}\right) \tilde{f}_{n}\left(\xi_{z}^{\prime}, \xi_{r}^{\prime}\right)\right] \xi_{r}^{\prime} \mathrm{d} \xi_{r}^{\prime} \\
& \times \int_{0}^{2 \pi} \sqrt{\left(\xi_{z}-\xi_{z}^{\prime}\right)^{2}+\xi_{r}^{2}+2 \xi_{r} \xi_{r}^{\prime} \cos \varphi+\xi_{r}^{\prime 2}} \mathrm{~d} \varphi,
\end{aligned}
$$

where $\varphi$ is the azimuth angle between vectors $\boldsymbol{\xi}$ and $\boldsymbol{\xi}^{\prime}$. One can approximate integrands in Eq. (37) by their values at the centers of cells with the second-order of accuracy: 


$$
\left(\widetilde{J}_{i n}\right)_{k l}=\widetilde{J}_{i n}\left[\left(\xi_{z}\right)_{k},\left(\xi_{r}\right)_{l}\right]=2 \frac{a}{\lambda_{s}} \frac{\sigma_{e x}}{\pi d^{2}}(1+w) \sum_{k^{*}, l^{*}=1}^{N_{z}, N_{r}}\left[\left(\tilde{f}_{i}\right)_{k^{*} l^{*}}\left(\tilde{f}_{n}\right)_{k l}-\left(\tilde{f}_{i}\right)_{k l}\left(\tilde{f}_{n}\right)_{k^{*} l^{*}}\right] g_{k l k^{*} l^{*} V_{l},}
$$

where the matrix of integrals

$$
g_{k l k^{*} l^{*}}=\frac{1}{\pi} \int_{0}^{\pi} \sqrt{\left[\left(\xi_{z}\right)_{k}-\left(\xi_{z}\right)_{k^{*}}\right]^{2}+\left(\xi_{r}\right)_{l}^{2}+2\left(\xi_{r}\right)_{l}\left(\xi_{r}\right)_{l^{*}} \cos \varphi+\left(\xi_{r}\right)_{l^{*}}^{2}} \mathrm{~d} \varphi,
$$

is numerically calculated once for a given velocity grid. Symmetry of this matrix about the exchange of index pairs $k l$ and $k^{*} l^{*}$ ensures conservation of mass.

Discrete velocity equation corresponding to kinetic equation for ions (28) is written as

$$
\begin{aligned}
(1 & +w) \frac{\partial\left(\tilde{f}_{i}\right)_{k l}}{\partial \tau}+\left(\xi_{z}\right)_{k} \frac{\partial\left(\tilde{f}_{i}\right)_{k l}}{\partial w}-\frac{1}{2 \widetilde{n}_{i}} \frac{\partial \widetilde{n}_{i}}{\partial w} \frac{\left(\tilde{f}_{i}\right)_{k+1 / 2, l}-\left(\tilde{f}_{i}\right)_{k-1 / 2, l}}{\Delta \xi_{z}} \\
& =\left(\tilde{J}_{i n}\right)_{k l},
\end{aligned}
$$

where the right-hand side is given by Eq. (38) and the conservativity of the advection term (last term in the left-hand side) is guaranteed by introducing values $\left(\widetilde{f}_{i}\right)_{k+1 / 2, l}$ of distribution function averaged over the boundary between cells $(k, l)$ and $(k+1, l)$. They are estimated by "minmod" slope limiter method: ${ }^{34}$

$$
\begin{aligned}
\left(\tilde{f}_{i}\right)_{k+1 / 2, l} & = \begin{cases}\Phi_{k}\left(\left(\xi_{z}\right)_{k}+\Delta \xi_{z} / 2\right), & \partial n_{i} / \partial w<0 \\
\Phi_{k+1}\left(\left(\xi_{z}\right)_{k}+\Delta \xi_{z} / 2\right), & \partial n_{i} / \partial w \geqslant 0\end{cases} \\
\Phi_{k}\left(\xi_{z}\right)= & \left(\tilde{f}_{i}\right)_{k l}+\frac{\xi_{z}-\left(\xi_{z}\right)_{k}}{\Delta \xi_{z}} \operatorname{minmod}\left(\left(\tilde{f}_{i}\right)_{k+1, l}-\left(\tilde{f}_{i}\right)_{k l},\left(\tilde{f}_{i}\right)_{k l}\right. \\
& \left.-\left(\tilde{f}_{i}\right)_{k-1, l}\right),
\end{aligned}
$$

with the "minmod" function

$$
\operatorname{minmod}(x, y)= \begin{cases}0, & x y \leqslant 0 \\ x, & x y>0 \text { and }|x|<|y| \\ y, & x y>0 \text { and }|x| \geqslant|y|\end{cases}
$$

This gives a second-order monotone approximation of the advection term. To specify reconstruction by Eq. (42) near boundaries $\xi_{z}=\Xi_{z}^{-}$and $\xi_{z}=\Xi_{z}^{+}$, ghost nodes are introduced with $\left(\widetilde{f}_{i}\right)_{-1 l}=\left(\widetilde{f}_{i}\right)_{0 l}=\left(\widetilde{f}_{i}\right)_{N_{z+1}, l}=\left(\widetilde{f}_{i}\right)_{N_{z}+2, l}=0$ for $l=1 \ldots N_{r}$.

\section{RESULTS AND DISCUSSION}

Numerical results are obtained for pressure ratios $\tilde{p}_{n}(\infty)$ from 0.22 to 0.8 and normalized charge exchange crosssection $\sigma_{e x} /\left(\pi d^{2}\right)$ from 2 to 10 . Transform parameter $a$ in Eq. (25) is set to $\lambda_{s} / 4$. The convergence of numerical solutions versus the number of grid points is estimated by comparison of test computations with different grid sizes. The optimal dimension of velocity grid is found to be $N_{z} \times N_{r}$ $=40 \times 20$. The optimal spatial grid size varies from 40 to 640 nodes. The higher spatial resolution is necessary for higher charge exchange cross-sections and lower pressure ratios.
Figure 4 shows profiles of distribution function moments and electrostatic potential $\phi$ in the Knudsen layer at evaporation with pressure ratio $\tilde{p}_{n}(\infty)=0.25$ and $\sigma_{e x} /\left(\pi d^{2}\right)=5$. At such pressure ratio, Mach number in the uniform vapor flow behind the Knudsen layer $M=0.857$ is obtained, where

$$
M=\frac{u_{n}(\infty)}{\sqrt{\frac{5}{3} \frac{k T_{n}(\infty)}{m_{n}}}}=\widetilde{u}_{n}(\infty) \sqrt{\frac{6}{5 \widetilde{T}_{n}(\infty)}} .
$$

Normalized electrostatic potential [see Fig. 4(a)] is obtained from Eqs. (9) and (10) as

$$
\frac{e \phi}{k T_{s}}=\ln \frac{\tilde{n}_{i}}{\tilde{n}_{i}(\infty)} .
$$

Charges do not influence neutrals according to the considered model. Therefore, neutral moments (broken lines in Fig. 4) are the same as at one-component evaporation calculated previously in Refs. 17 and 18. Boundary conditions (29) at the surface are equal for dimensionless distribution functions of ions and neutrals, so that differences between corresponding neutral and ion dimensionless moments in the Knudsen layer reflect difference in transport of these species described by Eqs. (26) and (28). In particular, the electrostatic force pushes ions in the direction from the surface that makes their flow velocity $\tilde{u}_{i}$ be greater than $\tilde{u}_{n}$ [see Fig. 4(b)] and dimensionless density $\tilde{n}_{i}>\widetilde{n}_{n}$ [Fig. 4(a)]. The difference in velocity decreases with the distance from the surface because the field becomes weaker and vanishes behind the Knudsen layer in the equilibrium vapor flow. On the contrary, the difference in density is accumulated downstream and remains constant behind the Knudsen layer.

Ionization degree in weakly ionized gas is generally defined as

$$
\alpha=\frac{n_{i}}{n_{n}}
$$

One can obtain it from dimensionless densities $\tilde{n}_{n}$ and $\tilde{n}_{i}$ given in Table II:

$$
\frac{\alpha}{\alpha}=\frac{1}{\sqrt{\tilde{n}_{i}(0)}} \frac{\tilde{n}_{i}}{\tilde{n}_{n}}
$$

Ionization degree normalized thus by its value in the saturated vapor $\alpha_{s}$ is considerably greater than unity as shown in Fig. 4(a). 

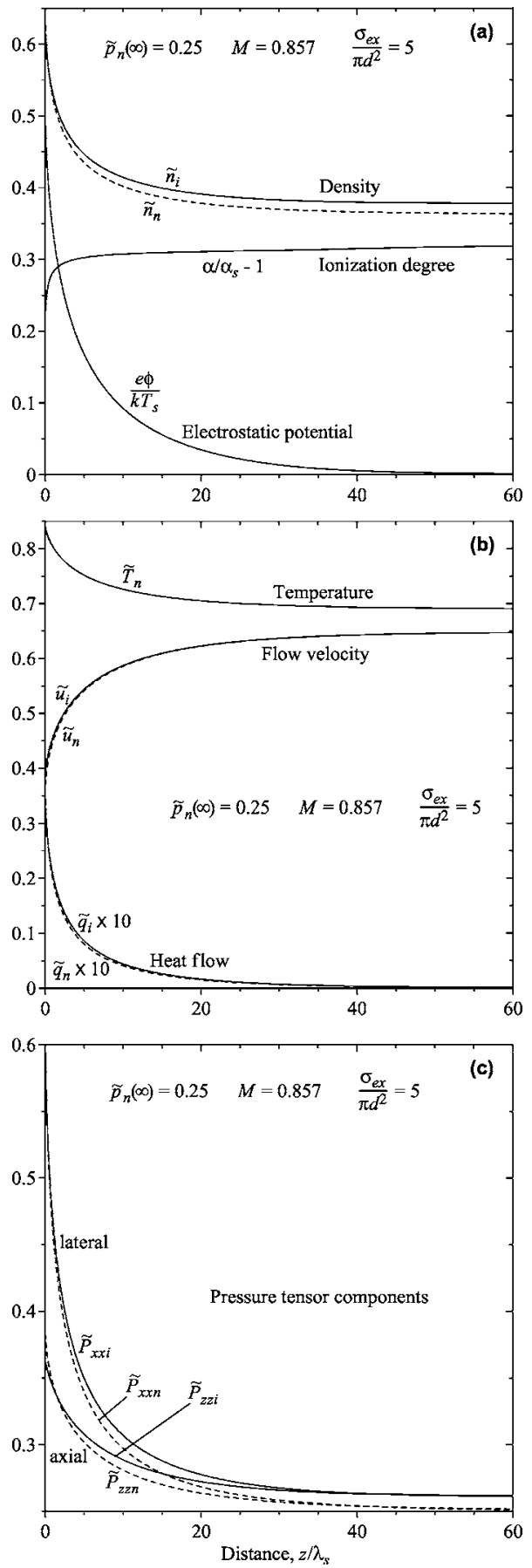

FIG. 4. Dimensionless parameters in the Knudsen layer versus distance $z$ from the condensed phase: (a) density $\widetilde{n}_{\beta}$, electrostatic potential $e \phi /\left(k T_{s}\right)$ and ionization degree $\alpha / \alpha_{s}$; (b) temperature $\widetilde{T}_{\beta}$, flow velocity $\widetilde{u}_{\beta}$, and heat flow $\widetilde{q}_{\beta}$; (c) axial, $\widetilde{P}_{z z \beta}$, and lateral, $\widetilde{P}_{x x \beta}=\widetilde{P}_{y y \beta}$, normal components of pressure tensor. Moments of velocity distribution function for neutrals (subscript $\beta=n)$ are shown by broken lines and the moments for ions $(\beta=i)$ are shown by full lines.

The difference between temperatures of neutrals $\widetilde{T}_{n}$ and ions $\widetilde{T}_{i}$ is small and not visible in Fig. 4(b). Heat flows, $\widetilde{q}_{n}$ and $\widetilde{q}_{i}$ [see Fig. 4(b)], and differences between lateral and axial normal components of pressure tensor, $\widetilde{P}_{x x n}-\widetilde{P}_{z z n}$ and $\widetilde{P}_{x x i}-\widetilde{P}_{z z i}$ [Fig. 4(c)], tend to zero with increasing the distance from the surface that indicates relaxation to equilib-
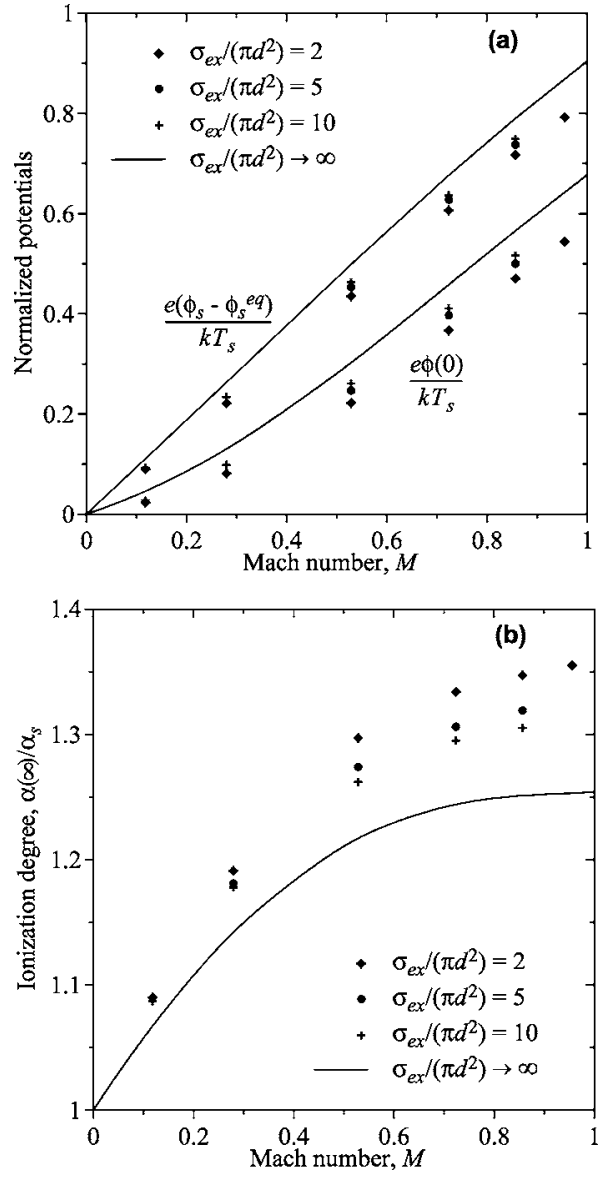

FIG. 5. Normalized condensed phase potential $e\left(\phi_{s}-\phi_{s}^{e q}\right) / k T_{s}[(\mathrm{a})$, upper curve], potential at the external boundary of the charge separation layer $e \phi(0) / k T_{s}$ [(a), lower curve], and ionization degree outside the Knudsen layer $\alpha(\infty) / \alpha_{s}$ (b) versus Mach number $M$. Points are obtained by numerical solution of kinetic equations for neutrals and ions at various normalized charge exchange cross-section $\sigma_{e x} /\left(\pi d^{2}\right)$. Curves are obtained by numerical solution of kinetic equations for neutrals and approximation $\widetilde{f}_{i}=\widetilde{f}_{n}$ for ions.

rium in the vapor flow. Notice that the values of dimensionless partial pressures $\widetilde{P}_{x x n}=\widetilde{P}_{z z n}$ and $\widetilde{P}_{x x i}=\widetilde{P}_{z z i}$ remain different in the equilibrium region. This corresponds to the difference in dimensionless densities of neutrals and ions.

Numerical calculations indicate that differences between corresponding dimensionless moments of neutrals and ions decrease with increasing charge-exchange cross-section $\sigma_{e x}$. Indeed, at high $\sigma_{e x}$, the charge-exchange integral (right-hand side) dominates in Eq. (28) that determines fast relaxation of velocity distribution of ions to that of neutrals. Therefore, one can expect that dimensionless distributions $\tilde{f}_{n}=\widetilde{f}_{i}$ become equal in the limit of very intensive charge-exchange interaction.

Figure 5 and Table III show numerical results obtained at various Mach numbers and charge-exchange cross-sections. Electrostatic potential $\phi(0)$ at the external boundary of the charge separation layer [three lower rows of points in Fig. 5(a)] is calculated by Eq. (45). At very high values of $\sigma_{e x}$, dimensionless density of ions $\widetilde{n}_{i}$ approaches that of neutrals $\tilde{n}_{n}$ [see Fig. 4(a)], and so this potential can be estimated from the density distribution of neutrals: 
TABLE III. Steady-state vapor parameters far from the condensed phase surface.

\begin{tabular}{|c|c|c|c|c|c|c|c|}
\hline \multicolumn{2}{|c|}{ Pressure ratio, $\widetilde{p}_{n}(\infty)$} & 0.8 & 0.6 & 0.4 & 0.3 & 0.25 & 0.22 \\
\hline \multicolumn{2}{|c|}{ Temperature ratio, $\widetilde{T}_{n}(\infty)$} & 0.953 & 0.891 & 0.801 & 0.734 & 0.689 & 0.657 \\
\hline \multicolumn{2}{|c|}{ Mach number, $M$} & 0.118 & 0.279 & 0.528 & 0.724 & 0.857 & 0.955 \\
\hline \multirow{4}{*}{$\begin{array}{l}\text { Ionization } \\
\text { degree, } \\
\alpha(\infty) / \alpha_{s}\end{array}$} & $\sigma_{e x} / \pi d^{2}=2$ & 1.090 & 1.191 & 1.297 & 1.334 & 1.347 & 1.355 \\
\hline & $\sigma_{e x} / \pi d^{2}=5$ & 1.088 & 1.181 & 1.274 & 1.306 & 1.319 & - \\
\hline & $\sigma_{e x} / \pi d^{2}=10$ & 1.087 & $\begin{array}{c}1.178 \\
\pm 0.002\end{array}$ & $\begin{array}{c}1.262 \\
\pm 0.002\end{array}$ & $\begin{array}{c}1.295 \\
\pm 0.005\end{array}$ & $\begin{array}{c}1.305 \\
\pm 0.005\end{array}$ & - \\
\hline & $\sigma_{e x} / \pi d^{2} \rightarrow \infty$ & 1.067 & 1.142 & 1.217 & 1.244 & 1.251 & 1.253 \\
\hline
\end{tabular}

$$
\frac{e \phi(0)}{k T_{s}}=\ln \frac{\tilde{n}_{n}(0)}{\tilde{n}_{n}(\infty)} .
$$

This approach is shown by the lower curve in Fig. 5(a). The value of $\phi(0)$ is always positive and increases with the Mach number. The electrostatic energy $e \phi(0)$ becomes comparable with the thermal energy $k T_{s}$ when the Mach number approaches unity. This indicates that the electrostatic field in the Knudsen layer significantly influences the distribution of charges (ions and electrons) at strong evaporation.

Electrostatic potential of the condensed phase relative to its equilibrium potential [three upper rows of points in Fig. $5(\mathrm{a})]$ is derived from Eqs. (23) and (45) as

$$
\frac{e\left(\phi_{s}-\phi_{s}^{e q}\right)}{k T_{s}}=\frac{1}{2} \ln \left[\tilde{n}_{i}(0)\right]-\ln \left[\tilde{n}_{i}(\infty)\right] .
$$

The relation between dimensional and dimensionless ion densities (see Table II) is taken into account to obtain Eq. (49). Substitution of $\widetilde{n}_{n}$ in place of $\widetilde{n}_{i}$ in Eq. (49) gives an estimate of the condensed phase potential at $\sigma_{e x} \rightarrow \infty$ [the upper curve in Fig. 5(a)]. The condensed phase potential increases approximately linearly with the Mach number $M$. It raises faster than $\phi(0)$, so that potential difference through the charge separation layer $\phi(0)-\phi_{s}>0$ decreases with $M$.

The results on vapor parameters in the uniform flow region outside the Knudsen layer are summarized in Table III. Pressure ratio $\tilde{p}_{n}(\infty)$ (first row) is specified and temperature ratio $\widetilde{T}_{n}(\infty)$, Mach number $M$, and ionization degree $\alpha(\infty)$ are calculated. The accuracy of the calculated values is up to the last digit written excluding cases where the error is given explicitly. The relations between $\tilde{p}_{n}(\infty), \widetilde{T}_{n}(\infty)$, and $M$ are the same as for neutral vapor. They were already discussed in Refs. 17 and 18 for the same collision model. The ionization degree is the original result obtained in this work. It is calculated by Eq. (47). In the limiting case $\sigma_{e x} \rightarrow \infty$ one can obtain:

$$
\frac{\alpha(\infty)}{\alpha_{s}}=\frac{1}{\sqrt{\tilde{n}_{n}(0)}} .
$$

Ionization degree $\alpha(\infty)$ is plotted versus Mach number $M$ in Fig. 5(b). It increases with $M$ up to about 1.3 of ionization in the saturated vapor $\alpha_{s}$ (remind that $\alpha_{s} \ll 1$ ). Ionization degree at the considered conditions is controlled by emission and transport of ions. The potential barrier in the charge separation layer near the condensed phase surface partly repels ejected ions. This barrier decreases with $M$ and allows more ions to pass through it. The contribution of this process to the ionization degree is given by the limiting curve at $\sigma_{e x} \rightarrow \infty$ in Fig. 5(b). Slipping of ions relative to neutrals becomes possible at finite values of $\sigma_{e x}$. As ions are forced by the electrostatic field in the Knudsen layer [see Fig. 4(a)], this gives an additional increase of ionization [points in Fig. 5(b)]. The contribution of the field in the Knudsen layer increases with decreasing $\sigma_{e x}$.

Figure 5(b) shows that ionization degree in vapor obtained by strong evaporation is greater than in the saturated vapor at the temperature of the condensed phase. Note that the temperature of the vapor is less than that of the condensed phase (see Table III), so that its equilibrium ionization should be still less than in the saturated vapor. Therefore, a considerable excess of charges is expected relative to the ionization equilibrium.

\section{CONCLUSIONS}

Strong evaporation of a condensed phase is considered at temperatures where its saturated vapor is weakly ionized. The Debye length is assumed to be much shorter than the mean free path, so that the plasma sheath consists of a Knudsen layer and a thin charge separation layer between the condensed phase and the Knudsen layer. The electrostatic potential of the condensed phase is assumed to be negative relative to the gas phase. These conditions are typical for laser evaporation of metals.

The transport of charges in the sheath is controlled by ions and depends on ion-neutral collisions and a selfconsistent electrostatic field. The principal interaction between ions and neutrals is the charge exchange. A potential barrier is formed in the charge separation layer that partly repels ions ejected from the condensed phase. Its absolute value decreases with the Mach number. An electrostatic field accelerating ions is formed in the Knudsen layer. The potential difference through the Knudsen layer increases with the Mach number.

Ionization degree in the vapor formed at strong evaporation increases with the Mach number and can attain values about 30\% higher than the ionization degree in the saturated vapor. Two factors contribute to this increase. The first is the drop of the potential barrier in the charge separation layer 
and the second is the strengthening of the field in the Knudsen layer. The ionization equilibrium may be disturbed by a considerable excess of charges.

\section{ACKNOWLEDGMENTS}

This work was performed while A.V.G. was staying in Kyoto University as a long-term fellow of the Japan Society for the Promotion of Science. A.V.G. thanks Professor Shigeru Takata for his kind hospitality.

${ }^{1}$ Ya. B. Zel'dovich, and Yu. P. Raiser, Physics of Shock Waves and HighTemperature Hydrodynamic Phenomena (Academic, New York, 1967).

${ }^{2}$ D. Breitling, H. Schittenhelm, P. Berger, F. Dausinger, and H. Hugel, Appl. Phys. A 69, S505 (1999).

${ }^{3}$ E. G. Gamaly, A. V. Rode, and B. Luther-Davies, J. Appl. Phys. 85, 4213 (1999).

${ }^{4}$ V. Yu. Fominski, V. N. Nevolin, R. I. Romanov, and I. Smurov, J. Appl. Phys. 89, 1449 (2001).

${ }^{5}$ V. I. Mazhukin, V. V. Nossov, M. G. Nickiforov, and I. Smurov, J. Appl. Phys. 93, 56 (2003).

${ }^{6}$ T. Tokonami and T. Makabe, J. Appl. Phys. 72, 3323 (1992).

${ }^{7}$ N. Sternberg and V. A. Godyak, Physica D 97, 498 (1996).

${ }^{8}$ K.-U. Riemann, Phys. Plasmas 4, 4158 (1997).

${ }^{9}$ R. N. Franklin and J. Snell, Phys. Plasmas 8, 643 (2001).

${ }^{10}$ A. V. Vasenkov and B. D. Shizgal, Phys. Plasmas 9, 691 (2002).

${ }^{11}$ A. V. Vasenkov and B. D. Shizgal, Phys. Rev. E 65, 046404 (2002).

${ }^{12}$ S. I. Anisimov, Sov. Phys. JETP 27, 182 (1968).

${ }^{13}$ T. Ytrehus, in Rarefied Gas Dynamics, edited by J. L. Potter (AIAA, New York, 1977), pp. 1197-1212.
${ }^{14}$ C. J. Knight, AIAA J. 17, 519 (1979).

${ }^{15}$ M. N. Kogan and N. K. Makashev, Fluid Dyn. 6, 913 (1971).

${ }^{16}$ D. Sibold and H. Urbassek, Phys. Fluids A 5, 243 (1993).

${ }^{17}$ Y. Sone and H. Sugimoto, in Adiabatic Waves in Liquid-Vapor Systems, edited by G. E. A. Meier and P. A. Thompson (Springer, Berlin, 1990), pp. 293-304.

${ }^{18}$ A. V. Gusarov and I. Smurov, Phys. Fluids 14, 4242 (2002).

${ }^{19}$ Y. Sone, Kinetic Theory and Fluid Dynamics (Birkhauser, Boston, 2002).

${ }^{20}$ Y. Sone, Transp. Theory Stat. Phys. 29, 227 (2000).

${ }^{21}$ Y. Sone, S. Takata, and F. Golse, Phys. Fluids 13, 324 (2001).

${ }^{22}$ A. V. Bobylev, R. Grzhibovskis, and A. Heintz, J. Stat. Phys. 102, 1151 (2001).

${ }^{23}$ A. Frezzotti, in Rarefied Gas Dynamics, edited by C. Shen (Peking University Press, Beijing, 1997), pp. 837-846.

${ }^{24}$ S. Takata, in Rarefied Gas Dynamics, edited by M. Capitelli (American Institute of Physics, Melville, 2005), pp. 503-508.

${ }^{25}$ Thermodynamic Properties of Substances, edited by V. P. Glushko (Nauka, Moscow, 1978).

${ }^{26}$ NIST-JANAF Thermochemical Tables, edited by M. W. Chase (National Institute of Standards and Technology, New York, 1998).

${ }^{27}$ S. Dushman, Rev. Mod. Phys. 2, 381 (1930).

${ }^{28}$ S. M. Sze, The Physics of Semiconductor Devices (Wiley, New York, 1981).

${ }^{29}$ D. E. Gray, American Institute of Physics Handbook (McGraw-Hill, New York, 1972)

${ }^{30}$ S. I. Braginskii, Sov. Phys. JETP 6, 358 (1958).

${ }^{31}$ B. M. Smirnov, Phys. Scr. 61, 595 (2000).

${ }^{32}$ L. Mieussens, J. Comput. Phys. 162, 429 (2000).

${ }^{33}$ L. D. Landau and E. M. Lifshitz, Statistical Physics (Pergamon, New York, 1980).

${ }^{34}$ R. J. Leveque, Numerical Methods for Conservation Laws (Birkhauser, Basel, 1992). 